\title{
Wavefront Correction Using Deformable Mirror for Extended Scene: Experimental Results
}

\author{
By Norihide MiYAmURA ${ }^{1)}$ and Nobutada SAKO ${ }^{2}$ \\ ${ }^{1)}$ Department of Interdisciplinary Science and Engineering, Meisei University, Tokyo, Japan \\ ${ }^{2)}$ Canon Electronics Inc., Tokyo, Japan
}

(Received June 30th, 2017)

\begin{abstract}
We are developing an image based adaptive optics system using a micro electro mechanical system (MEMS) deformable mirror for earth observing sensors. We proposed two algorithms for control of the deformable mirror, a stochastic parallel gradient descent (SPGD) optimization and a deformable mirror diversity estimation. We developed an adaptive optics system testbed using a commercial astronomical telescope and a MEMS deformable mirror. We observed a distant building pattern from our laboratory, then controlled the deformable mirror to compensate the wavefront aberration. Experimental results show that misalignments and wavefront aberrations are compensated and image qualities are improved.
\end{abstract}

Key Words: Adaptive Optics, Deformable Mirror, Remote Sensing

Nomenclature

$\begin{array}{lll}a & : & \text { wavefront aberration coefficient } \\ b & : \text { phase diversity coefficient } \\ i & : & \text { observed image } \\ J & : & \text { merit function of observed image } \\ s & : & \text { point spread function } \\ n & : & \text { image noise } \\ o & : & \text { object } \\ P & : \text { pupil function } \\ P_{g} & : \text { generalized pupil function } \\ r & : \text { random sign of the perturbation } \\ u & : \text { input for deformable mirror actuator } \\ \left(f_{x}, f_{y}\right) & : \text { spatial frequency plane coordinate } \\ (x, y) & : \text { focal plane coordinates } \\ z_{f} & : \text { focal length } \\ Z & : \text { Zernike polynomials } \\ \lambda & : \text { wavelength } \\ \gamma & : \text { feedback gain of SPGD method } \\ \Phi & : \text { wavefront function } \\ \Theta & : \text { phase diversity wavefront function } \\ \mu & : \text { average in the specific image area } \\ \sigma & : \text { standard deviation in the specific } \\ (\xi, \eta) & : \text { image area } \\ & \text { pupil plane coordinate }\end{array}$

Subscripts
(k) : $\quad k$ th deformable mirror diversity
$j \quad: \quad j$ th Zernike polynomial
$m \quad: \quad m$ th trial in SPGD method
$n \quad: \quad$ actuator number of the deformable mirror

\section{Introduction}

A high-resolution observation is required for small satellite remote sensing missions in recent years. A large aperture telescope of more than $400 \mathrm{~mm}$ aperture should be used to realize less than $1 \mathrm{~m}$ GSD observations. However, it is difficult or expensive to realize the large aperture telescope using a monolithic primary mirror with high surface accuracy. A segmented mirror telescope or a deployable telescope should be studied especially for small satellite missions. Generally, not only high accuracy of optical surface but also high accuracy of optical alignment is required for large aperture telescopes. For deployable telescopes, alignments of optical elements are more difficult than that of conventional monolithic mirror telescopes. For conventional systems, optical alignments are adjusted before launch to achieve desired imaging performance. However, it is difficult to adjust the alignment for large sized optics in high accuracy. Furthermore, thermal environment in orbit and vibration in a launch vehicle cause the misalignments of the optics. We are developing an adaptive optics system ${ }^{1,2)}$ using a micro electro mechanical system (MEMS) deformable mirror ${ }^{3-5)}$ for earth observing remote sensing sensors that can be equipped on small satellites. An image based adaptive optics system ${ }^{6-11)}$ compensates misalignments of optical elements and wavefront aberrations of optics using a MEMS deformable mirror by feedback control using observed images instead of using a wavefront sensor. ${ }^{12)}$ By using the image based adaptive optics, total amount of incident light can enter an image sensor. We developed an adaptive optics system testbed using a commercial astronomical telescope and a deformable mirror. We observed a distant building pattern from our laboratory, then controlled the deformable mirror to compensate the intentional misalignment of the optical system. We propose the control algorithm of the deformable mirror for extended 
scenes by using observed images, then present experimental results for several modes of wavefront aberrations that are intentionally added by the deformable mirror. Experimental results show that misalignments and wavefront aberrations are compensated and the image quality is improved.

\section{Mathematical Model of the Adaptive Optics System and the Wavefront Aberration}

The observed image is calculated by convolution of the object and the point spread function (PSF) as Eq. (1) based on the scholar diffraction theorem, ${ }^{13)}$

$$
i(x, y)=s(x, y) * o(x, y)+n(x, y) .
$$

The aberration of the optics affects the shape of the PSF which is calculated by the Fourier transform of the generalized pupil function as Eq. (2),

$$
s(x, y)=\left\{F\left[P_{g}\left(\frac{x}{\lambda z_{f}}, \frac{y}{\lambda z_{f}}\right)\right]\right\}^{2},
$$

where

$$
P_{g}(\xi, \eta)=P(\xi, \eta) \exp \{i \Phi(\xi, \eta)\} .
$$

The generalized pupil function is calculated using the wavefront function and the pupil function. The wavefront function is expressed by a linear combination of Zernike polynomials $^{14)}$ as Eq. (4).

$$
\Phi(\xi, \eta)=\sum_{j=1}^{J} a_{j} Z_{j}(\xi, \eta),
$$

Low order Zernike polynomials are shown in Table 1. These modes are correlated with Seidel aberrations.

Table 1. Aberration modes of Zernike polynomials.

\begin{tabular}{lll}
\hline Term & Zernike polynomials & Seidel \\
\hline 1 & 1 & piston \\
2 & $2 r \cos \theta$ & x tilt \\
3 & $2 r \sin \theta$ & $y$ tilt \\
4 & $\sqrt{3}\left(2 r^{2}-1\right)$ & defocus \\
5 & $\sqrt{6} r^{2} \sin 2 \theta$ & y primary astigmatism \\
6 & $\sqrt{6} r^{2} \cos 2 \theta$ & x primary astigmatism \\
7 & $\sqrt{8}\left(3 r^{3}-2 r\right) \sin \theta$ & y primary coma \\
8 & $\sqrt{8}\left(3 r^{3}-2 r\right) \cos \theta$ & x primary coma \\
9 & $\sqrt{8} r^{3} \sin 3 \theta$ & y trefoil \\
10 & $\sqrt{8} r^{3} \cos 3 \theta$ & z trefoil \\
\hline
\end{tabular}

\section{Optimization Algorithm for Compensation of Wavefront Error}

\subsection{Stochastic parallel gradient descent optimization using a deformable mirror}

In stochastic parallel gradient descent (SPGD) optimization using a deformable mirror, stochastic inputs are applied to wavefronts using a deformable mirror, then the merit function calculated from the observed image is optimized. To determine $u_{n}^{m+1}, u_{n}^{m+}$ and $u_{n}^{m-}$ are calculated as Eqs. (5-6) and are applied to the deformable mirror to obtain images. The merit function $J^{m+}$ and $J^{m-}$ are calculated for each image. Then $\delta J^{m}$ is calculated as Eq. (7). Finally, $u_{n}^{m+1}$ is calculated as Eq. (8) then applied to the deformable mirror. The value of +1 or -1 is randomly assigned to $r_{n}^{m}$. The value of the perturbation $\delta u$ for examining the change of the merit function $J^{m+}$ and $J^{m-}$ was set to 0.1 , and the feedback gain $\gamma$ was set to 0.2 . These values were determined by trial and error tuning. The sequence of steps is iterated until the change in the merit function becomes sufficiently small.

$$
\begin{gathered}
u_{n}^{m+}=u_{n}^{m}+r_{n}^{m} \delta u, \\
u_{n}^{m-}=u_{n}^{m}-r_{n}^{m} \delta u, \\
\delta J^{m}=J^{m+}-J^{m-}, \\
u_{n}^{m+1}=u_{n}^{m}+\gamma \delta J^{m} r_{n}^{m} \delta u,
\end{gathered}
$$

In this method, the merit function $J$ perform an important role for the SPGD optimization. We considered two merit functions. One is based on the sharpness of the image, and the other is based on the contrast of the image.

The sharpness merit function is expressed as Eq. (9).

$$
J_{s}=\int\{i(x, y)\}^{2} d x d y .
$$

When an optical system has a wavefront aberration, the PSF spreads broadly, then energy of the incident light decreases. Therefore, the image could be sharpened by maximize the pixel value of the image using the merit function $J_{s}$.

The contrast merit function is calculated as Eq. (10),

$$
J_{c}=\frac{\sigma}{\mu} .
$$

This merit function reflects the difference of the brightness of the image in the specific area. When the PSF spreads broadly, observed image blurs and the difference between the brightness of pixels becomes smaller. Therefore, the image contrast could be improved by maximize the contrast of the image.

The sharpness merit function has a high sensitivity against the Zernike coefficients for a point source target such as a star. On the other hand, when observing an extended scene, the sensitivity of this merit function against the Zernike coefficients decrease considerably. This is because pixels in the neighboring region affect each other due to blur. Fig. 1 shows the relationship between the value of the Zernike coefficient $a_{4}$ and values of merit functions when observing a extended scene. When the absolute value of the $a_{4}$ becomes larger, the sensitivity of the $J_{\mathrm{s}}$ decreases compared with that of $J_{\mathrm{c}}$. Therefore, we use the $J_{\mathrm{c}}$ for an extended scene. 


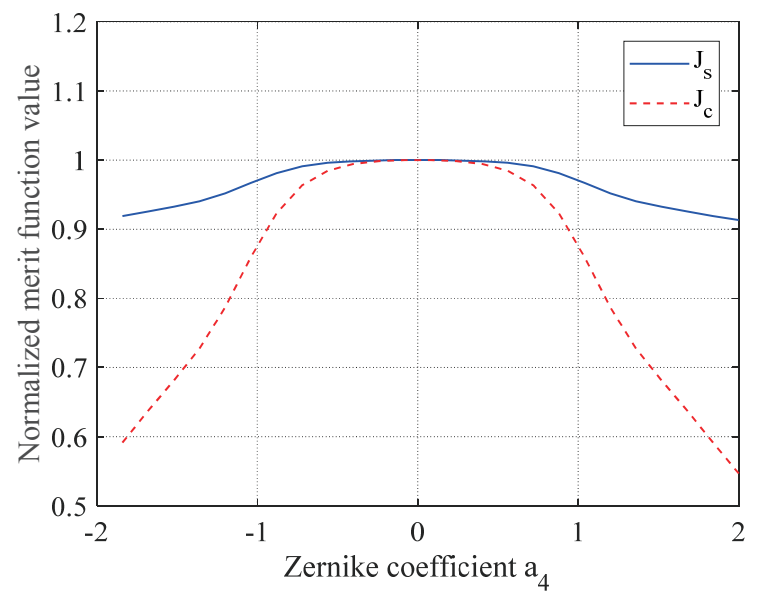

Fig. 1. The relationship between Zernike coefficient $a_{4}$ and the values of merit functions.

Numerical simulation results of the SPGD optimization are shown in Figs. 2 and 3. For the wavefront aberration, Zernike coefficients of 10 modes shown in Table 1 were randomly generated with the standard deviation of 0.5 and the average of 0.3 . The wavefront aberration is compensated by optimizing the contrast merit function.

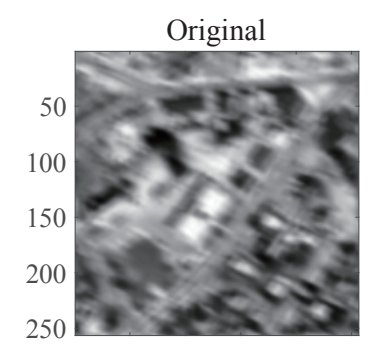

$50 \quad 100 \quad 150 \quad 200 \quad 250$

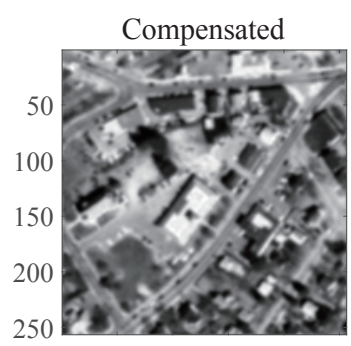

$\begin{array}{lllll}50 & 100 & 150 & 200 & 250\end{array}$
Fig. 2. Numerical simulation results of the SPGD optimization using the contrast merit function. The original image (left), and the compensated image (right) are shown.

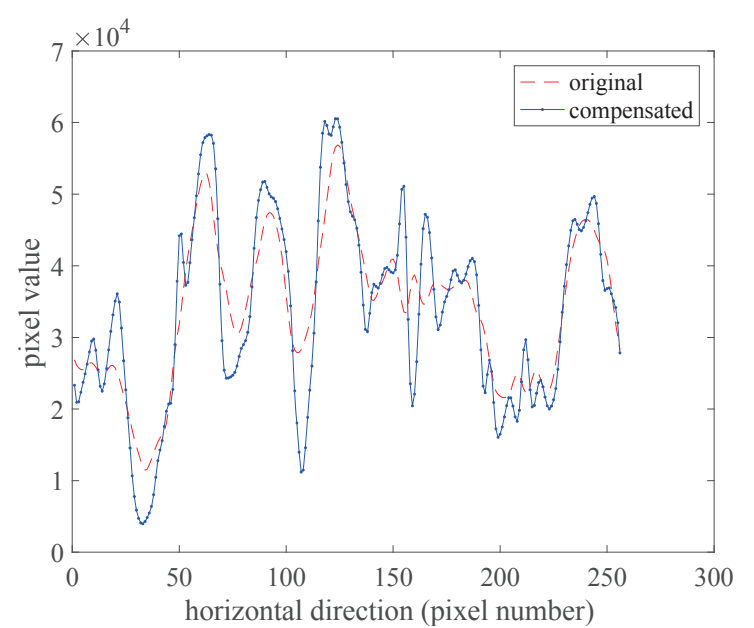

Fig. 3. Pixel values of numerical simulation results of the SPGD optimization.
In implementing the SPGD optimization to the adaptive optics system, the merit function must be calculated from the obtained image. In other words, it is necessary to implement the wavefront compensation software on the on-board computer and to control the deformable mirror online.

On the other hand, the SPGD optimization does not require a mathematical model which represents the relationship between input voltages of the deformable mirror and obtained images. Therefore, the SPGD optimization can be regarded as a robust method against structural changes such as thermal distortions or misalignments.

\subsection{Deformable mirror diversity estimation}

In the phase diversity wavefront estimation, the wavefront aberration whose size is known, called the phase diversity, must be intentionally added. In the conventional method, the phase diversity is generated by defocus of an image sensor. An estimation problem of the wavefront aberration is formulated as an inverse problem using multiple images with different phase diversities. We use a deformable mirror to apply phase diversities to optical wavefronts instead of defocusing the image sensor. Therefore, we call this method the deformable mirror diversity estimation. When the phase diversity is applied to the wavefront by the deformable mirror, the observed image is obtained as Eq. (11),

$$
i^{(k)}(x, y)=s^{(k)}(x, y) * o(x, y)+n^{(k)}(x, y) .
$$

The PSF of the optics with the deformable mirror diversity is calculated as Eq. (12),

$$
s^{(k)}(x, y)=\left\{F\left[P_{g}^{(k)}\left(\frac{x}{\lambda z_{f}}, \frac{y}{\lambda z_{f}}\right)\right]\right\}^{2},
$$

where

$$
P_{g}^{(k)}(\xi, \eta)=P(\xi, \eta) \exp \left\{i\left(\Phi(\xi, \eta)+\Theta^{(k)}(\xi, \eta)\right)\right\} .
$$

The wavefront aberration is sum of $\Phi(\xi, \eta)$ and $\Theta^{(k)}(\xi, \eta)$ that are given as Eqs. (14-15),

$$
\begin{gathered}
\Phi(\xi, \eta)=\sum_{j=1}^{J} a_{j} Z_{j}(\xi, \eta), \\
\Theta^{(k)}(\xi, \eta)=\sum_{j=1}^{J} b_{j}^{(k)} Z_{j}(\xi, \eta) .
\end{gathered}
$$

In the deformable mirror diversity estimation, aberration parameters, coefficients of Zernike polynomials, are estimated by minimizing the evaluation function $J_{D}{ }^{9)}$ as Eq. (16),

$$
J_{D}=\sum_{f_{x}} \sum_{f_{y}} \frac{\left|\sum_{k=1}^{K} I_{k}\left(f_{x}, f_{y}\right) H_{k}^{*}\left(f_{x}, f_{y}\right)\right|^{2}}{\sum_{k=1}^{K}\left|H_{k}\left(f_{x}, f_{y}\right)\right|^{2}},
$$

where $I$ and $H$ are the Fourier transform of $i$ and $h$ respectively and an asterisk used as a superscript implies complex conjugation. The merit function in the deformable mirror diversity estimation reflects characteristics of the PSF using coefficients of Zernike polynomials. Therefore, in this 
method, a mathematical model of the optical system including a shape of the deformable mirror surface is required.

Numerical simulation results of the deformable mirror diversity estimation are shown in Figs. 4 and 5. Conditions of the aberration were the same as those of the SPGD optimization. That is, coefficients of 10 Zernike polynomials were randomly generated with the standard deviation of 0.5 and the average of 0.3 . The optical wavefront was compensated using the estimated values of Zernike coefficients, then the observed image was calculated as shown in Fig. 4.

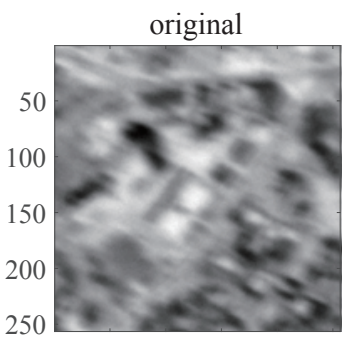

$50 \quad 100 \quad 150 \quad 200 \quad 250$

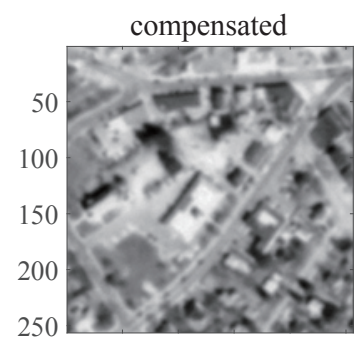

$\begin{array}{lllll}50 & 100 & 150 & 200 & 250\end{array}$
Fig. 4. Numerical simulation results of the deformable mirror diversity estimation. The original image (left), and the compensated image (right) are shown.

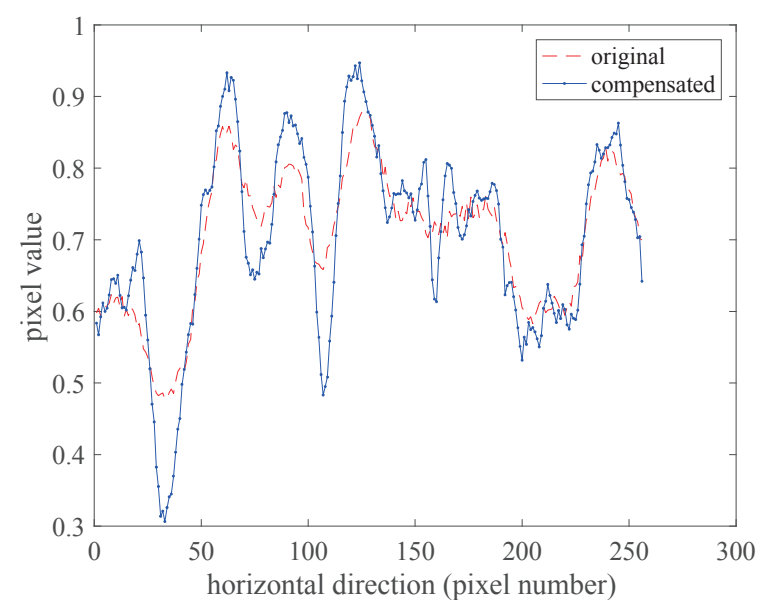

Fig. 5. Pixel values of numerical simulation results of SPGD method.

In the deformable mirror diversity method, the wavefront can be estimated by batch processing using the acquired image and the shape of the deformable mirror which was applied in acquiring images. After estimating the wavefront aberration, wavefront compensation is performed by giving an opposite phase with the deformable mirror. In other words, it is possible not to mount algorithms on the onboard computer and to process them offline.

On the other hand, when constructing the inverse problem, it is necessary to know deformable mirror diversities correctly, and it is important to construct a mathematical model of the deformable mirror and the optical system.

\section{Adaptive Optics Experimental Setup}

We developed the adaptive optics experimental system on the optical bench as Figs. 6 and 7. We used a commercial astronomical telescope with $120 \mathrm{~mm}$ aperture and $1000 \mathrm{~mm}$ focal length. A parallel light was guided to the deformable mirror by an afocal system, and the modulated wavefront was focused on a CMOS sensor by the condensing optical system.

The appearance of the system is shown in Fig. 7. We adjusted an alignment of the adaptive optics system with the deformable mirror on the optical breadboard in advance. Then, the alignment between the astronomical telescope and the adaptive optical system was adjusted on the optical bench.

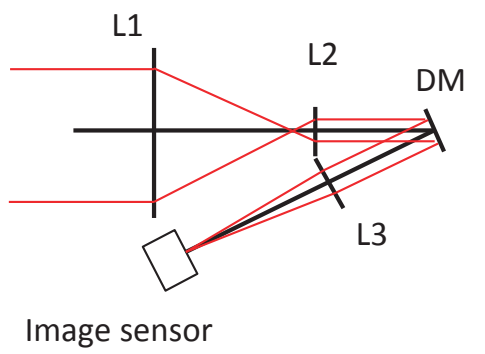

Fig. 6. A schematic of the optical layout of the adaptive optics experiment system.

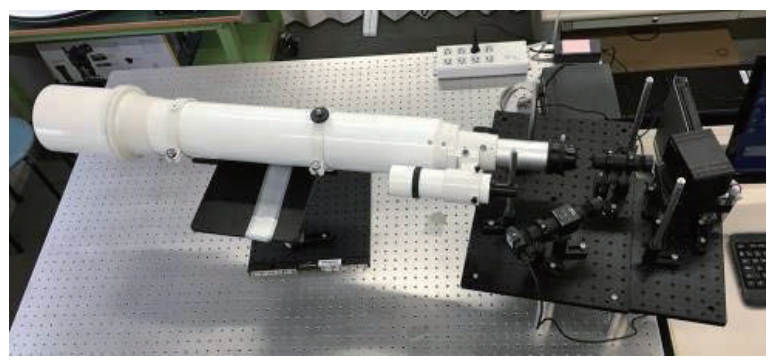

Fig. 7. Appearance of the adaptive optics experimental system.

We use a 140-actuator micro electro mechanical system (MEMS) deformable mirror manufactured by Boston Micromachines Corporation (BMC) shown in Fig. 8 for a wavefront modulation. The main specifications of the deformable mirror are shown in Table 2.

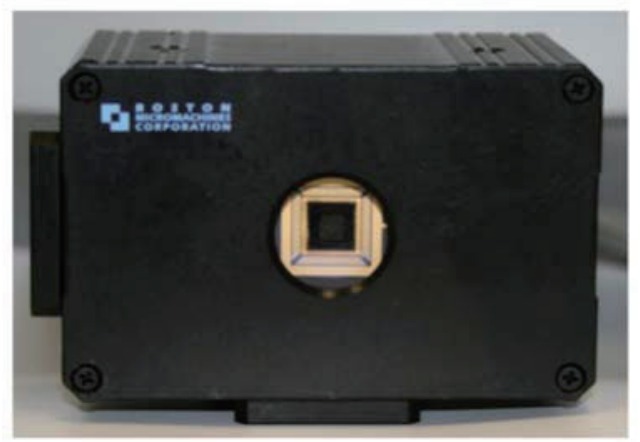

Fig. 8. BMC's 140-actuator MEMS deformable mirror. 
Table 2. Specifications of the MEMS deformable mirror.

\begin{tabular}{ll}
\hline Actuators & 140 \\
Stroke & $3.5 \mu \mathrm{m}$ \\
Aperture & $4.4 \mathrm{~mm} \times 4.4 \mathrm{~mm}$ \\
Pitch & $400 \mu \mathrm{m}$ \\
Response time $(10 \%$ to $90 \%)$ & $<100 \mu \mathrm{s}$ \\
Coupling & $13 \%$ \\
Coating & Aluminum \\
Cover glass & $350 \mathrm{~nm}$ to $700 \mathrm{~nm}$ \\
Fill factor & $>99 \%$ \\
\hline
\end{tabular}

We observed a pattern of the building $3 \mathrm{~km}$ away from our laboratory. The observed target is shown in Fig. 9 which was observed using a 400-mm aperture telescope for reference.

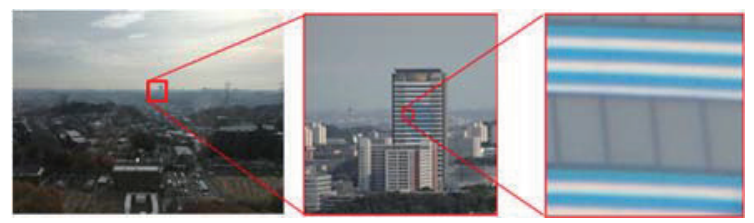

Fig. 9. The pattern of the building observed in the experiment.

\section{Experimental Results}

We focused on the fact that it is not necessary to construct a mathematical model of the optical system and the deformable mirror, therefore, we conducted adaptive optics experiments using the SPGD optimization described in 3.1. In this experiment, the contrast merit function was used. Both an unknown wavefront aberration inputs DM_abr and a wavefront control inputs DM_ctr were generated by the deformable mirror. That is, the input pattern of the deformable mirror is represented as DM abr+DM ctr. Then the amount of DM_ctr was optimized using the SPGD optimization. The initial value of the DM_ctr was set to random value with zero as the average value.

Experimental results for two different random values of DM_abr are shown below. The results of the experiment 1 are shown in Figs. 10 to 12, and those of the experiment 2 are shown in Figs. 13 to 15. In both cases, it can be confirmed that inputs distribution of the deformable mirror actuators became almost flat and the image quality was improved. The result means that DM_ctr could successfully cancel the unknown pattern of DM_abr.

In the experiment 1, the pattern of DM_abr is shown in the left of Fig. 10, and the compensated inputs pattern of the deformable mirror is shown in the right of Fig. 10.

The blurred pattern of the building was compensated by the SPGD optimization with the deformable mirror as shown in Fig. 11. The relationship between number of trials and the values of merit function in the experiment 1 is shown in Fig. 12. In this case, the merit function was minimized after 24 trials.
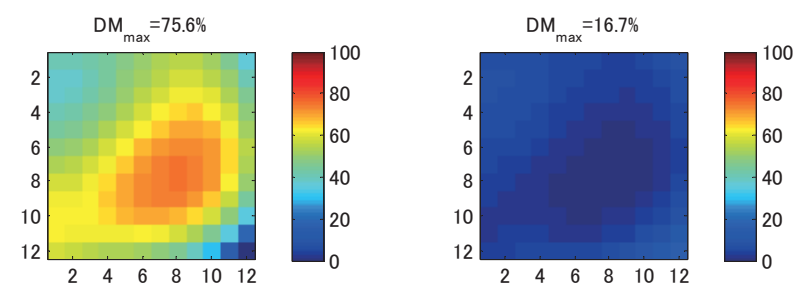

Fig. 10. Inputs for the actuators of the deformable mirror in Experiment 1. DM_init (left) and DM_init+x_opt (left)
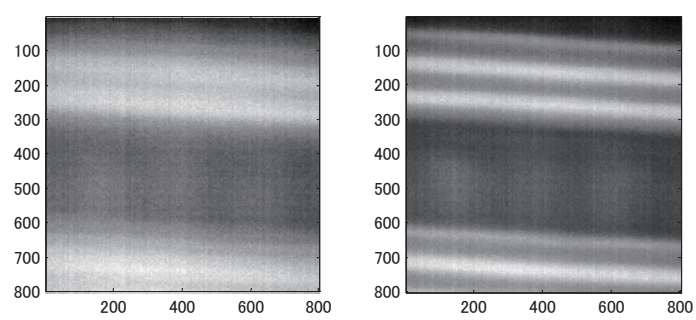

Fig. 11. Images in Experiment 1. DM_init (left) and DM_init $+x \_o p t$ (left)

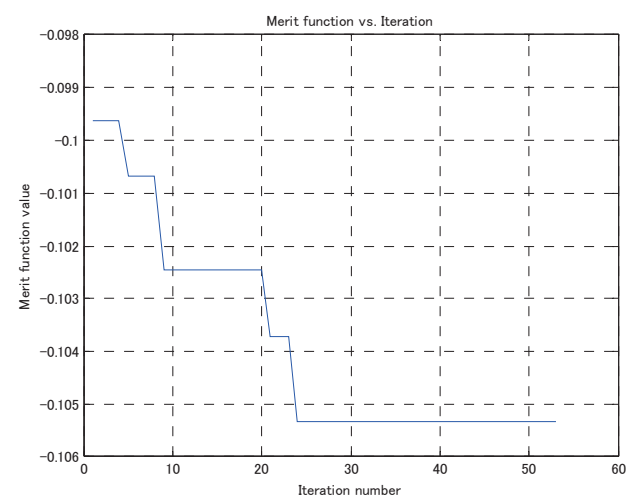

Fig. 12. Relationship between number of trials and the value of merit function in the experiment 1 .

In the experiment 2, the different pattern of DM_abr was randomly generated as shown in the left Fig. 13, and the compensated input pattern of the deformable mirror is shown in the right of Fig. 13.

As in the experiment 1 , the blurred pattern of the building was compensated by the SPGD optimization with the deformable mirror in the experiment 2 as shown in Fig. 14. The relationship between number of trials and the values of merit function in experiment 2 is shown in Fig. 15. In this case, the merit function was almost minimized after 4 trials.
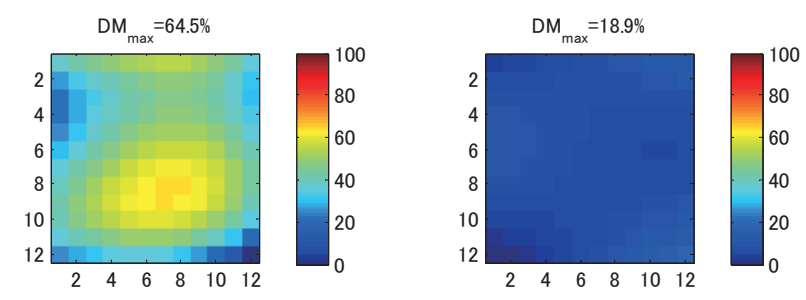

Fig. 13. Inputs for the actuators of the deformable mirror in Experiment 2. DM_init (left) and DM_init+x_opt (left) 

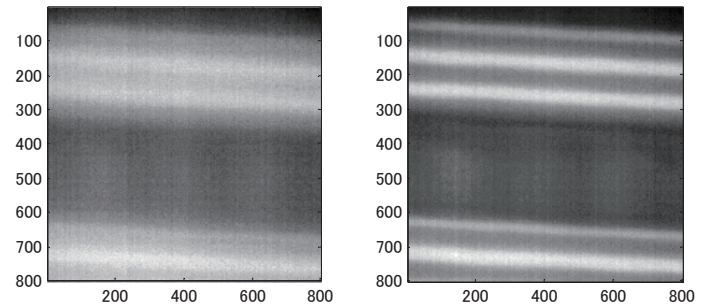

Fig. 14. Images in Experiment 2. DM_init (left) and DM_init + x_opt (left)

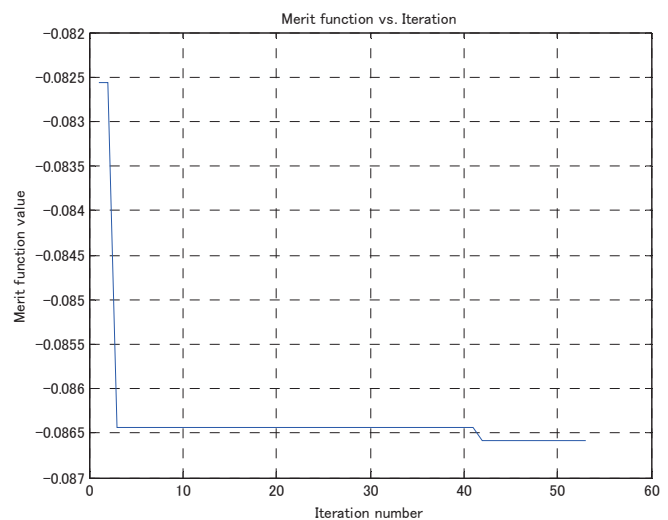

Fig. 15. Relationship between number of trials and the value of merit function in the experiment 2 .

\section{Conclusion}

We proposed two wavefront compensation methods, the SPGD optimization and the deformable mirror diversity estimation, for an adaptive optics system with a deformable mirror. Then we carried out numerical simulations for both methods, and it was shown that the wavefront aberration can be estimated using observed images by using the deformable mirror.

Furthermore, we constructed an adaptive optics experiment system using a MEMS deformable mirror and conducted experiments to observe a scene from our laboratory. As a result, we showed that the unknown wavefront aberration was canceled by the MEMS deformable mirror using the SPGD optimization. the image quality was improved.

\section{Acknowledgments}

This work was supported by Canon electronics Inc.

\section{References}

1) Tyson, R.: Principles of Adaptive Optics, CRC Press, 2011.

2) Hardy, J. W., Lefebvre, J. E. and Koliopoulos, C. L.: Real-time atmospheric compensation, J. Opt. Soc. Am., 67(3), (1977), pp. $360-369$.

3) Freeman, R. H. and Pearson, J. E.: Deformable mirrors for all seasons and reasons, Appl. Opt., 21(4) (1982), pp. 580-588.

4) Bifano, T., Bierden, P. and Perreault, J.: Micromachined deformable mirrors for dynamic wavefront control, Proc. SPIE, 5553 (2004), pp. 1-16.

5) Cornelissen, S. A.: 4096-element continuous face-sheet MEMS deformable mirror for high-contrast imaging, J. Micro/Nanolith. MEMS MOEMS, 8(3) (2009), pp. 031308-1-031308-8.

6) Miyamura, N.: Generalized phase diversity method for self-compensation of wavefront aberration using spatial light modulator, Opt. Eng., 48(12) (2009), pp. 128201-1-128201-8.

7) Gonsalves, R. A.: Phase retrieval and diversity in adaptive optics, Opt. Eng., 21(5) (1982), pp. 829-832.

8) Paxman, R. G., Schulz, T. J. and Fienup, J. R.: Joint estimation of object and aberrations by using phase diversity, J. Opt. Soc. Am. A, 9(7) (1992), pp. 1072-1085.

9) Vorontsov, M. A.: Decoupled stochastic parallel gradient descent optimization for adaptive optics: integrated approach for wave-front sensor information fusion, J. Opt. Soc. Am. A, 19(2) (2002), pp. 356-368.

10) Yang, H., Soloviev, O. and Verhaegen, M.: Model-based wavefront sensorless adaptive optics system for large aberrations and extended objects, Opt. Express, 23(19) (2015), pp. 24587-24601.

11) Gonsalves, R. A.: Sequential diversity imaging of extended sources, Proc. SPIE, 4792 (2002), pp. 26-34.

12) Védrenne, N. et al.: Shack-Hartmann wavefront estimation with extended sources: anisoplanatism influence, J. Opt. Soc. Am. A, 24(9) (2007), pp. 2980-2993.

13) Goodman, J. W.: Introduction to Fourier Optics 3rd edition, Roberts and Company, 2005.

14) Noll, R. J.: Zernike polynomials and atmospheric turbulence, $J$. Opt. Soc. Am., 66(3) (1976), pp. 207-211. 